\title{
Interpretaciones del Signo Igual en un Contexto Algebraico de Polinomios
}

\section{Interpretations of the Equal Sign in an Algebraic Polynomial Context}

\author{
Sebastián Parodi* \\ ORCID iD 0000-0002-9663-3990 \\ Cristina Ochoviet ${ }^{* *}$ \\ ORCID iD 0000-0001-9069-3469 \\ Javier Lezama*** \\ ORCID iD 0000-0002-9942-6428
}

\begin{abstract}
Resumen
Se presenta un estudio de casos que explora las interpretaciones del signo igual de un grupo de estudiantes de enseñanza media de Uruguay (13-14 años) en un contexto algebraico de polinomios. Se adopta como perspectiva teórica una clasificación de los significados del signo igual. Se aplica un cuestionario y se realizan entrevistas. Los resultados muestran que un conocimiento sobre el signo igual facilita el entendimiento de los polinomios y que el trabajo con los polinomios enriquece la comprensión del signo igual. Esto deja entrever una relación dialéctica entre la interpretación del signo igual y el aprendizaje de los polinomios. Implicaciones didácticas son propuestas a partir de estos hallazgos.
\end{abstract}

Palabras clave: Signo igual. Polinomios. Pensamiento relacional. Enseñanza media.

\begin{abstract}
We present a case study that explores the interpretations of the equal sign by a group of middle school students in Uruguay (13-14 years old) in an algebraic polynomial context. As a theoretical perspective, we adopted a classification of the equal sign meanings. We applied a questionnaire and performed interviews. The results show that knowledge about the equal sign facilitates the understanding of polynomials and that working with polynomials enriches the understanding of the equal sign. This suggests a dialectical relationship between the interpretation of the equal sign and the learning of polynomials. Didactic implications are proposed based on these findings.
\end{abstract}

\footnotetext{
* Magíster en Matemática Educativa por el Centro de Investigación en Ciencia Aplicada y Tecnología Avanzada del Instituto Politécnico Nacional (CICATA-IPN). Profesor de Matemática en el Consejo de Formación en Educación (CFE) y en el Consejo de Educación Secundaria (CES), Montevideo, Uruguay. Dirección postal: Asilo 3255, Unión, 11600, Montevideo, Uruguay. E-mail: parodiseb@gmail.com.

** Doctora en Matemática Educativa por el Centro de Investigación en Ciencia Aplicada y Tecnología Avanzada del Instituto Politécnico Nacional (CICATA-IPN). Coordinadora Académica del Departamento de Matemática del Consejo de Formación en Educación (CFE), Montevideo, Uruguay. Dirección postal: Asilo 3255, Unión, 11600, Montevideo, Uruguay. E-mail: cristinaochoviet@gmail.com.

**** Doctor en Matemática Educativa por el Centro de Investigación y de Estudios Avanzados del Instituto Politécnico Nacional (CINVESTAV-IPN). Profesor-investigador en el Programa de Matemática Educativa del Centro de Investigación en Ciencia Aplicada y Tecnología Avanzada del Instituto Politécnico Nacional (CICATAIPN), Ciudad de México, México. Dirección postal: Legaria 694, Irrigación, Miguel Hidalgo, 11500, Ciudad de México, México. E-mail: jlezamaipn@gmail.com.
} 
Keywords: Equal sign. Polynomials. Relational thinking. Middle school.

1 Introducción

En este trabajo, el álgebra es concebida como una herramienta que posibilita el estudio de patrones, relaciones y funciones; así como el análisis de las estructuras subyacentes a los cálculos y las relaciones; la modelización de relaciones cuantitativas y el estudio del cambio (NCTM, 2008). Esta manera de concebir el álgebra admite, pero no exige, el uso de letras para expresar y manipular las cantidades desconocidas (RADFORD, 2011, p. 310):

El pensamiento algebraico no tiene que ver con usar notación, sino con razonar de
determinadas maneras. Pensar en forma algebraica implica manejar las cantidades
indeterminadas de formas analíticas. Es decir, usted considera las cantidades
indeterminadas (las incógnitas o las variables) como si fueran conocidas y realiza
cálculos con estas como si fueran números conocidos.

Desde esta perspectiva, varios estudios realizados a nivel nacional e internacional reportan una tendencia de los estudiantes de enseñanza media a interpretar el signo igual como el indicador del resultado de una operación y no como el indicador de una relación de equivalencia, que es una interpretación necesaria para el estudio del álgebra así concebida (KIERAN, 1992; BURGELL; OCHOVIET, 2015, entre otros). Estos trabajos, no solamente exploran los significados que los alumnos le atribuyen al signo igual, sino que advierten sobre las dificultades que estas interpretaciones pueden ocasionar en los estudiantes, por ejemplo, al incursionar en el aprendizaje de las ecuaciones (KNUTH et al., 2011; PARODI; OCHOVIET; LEZAMA, 2017, entre otros).

En este reporte, a partir de un estudio que explora las interpretaciones del signo igual que evidencia un grupo de estudiantes de segundo año de enseñanza media de Uruguay (1314 años) en un contexto algebraico de polinomios (PARODI, 2016), se propone discutir el análisis en profundidad de dos casos, con el objetivo de responder la siguiente pregunta: ¿qué relación existe entre los significados que se le atribuyen explícitamente al signo igual y el trabajo con este signo en un contexto algebraico de polinomios? Los hallazgos obtenidos, contribuirán al conocimiento matemático para la enseñanza y aportarán insumos para pensar la enseñanza del álgebra en el ámbito de la enseñanza media.

\section{Antecedentes}

Una amplia cantidad de trabajos analizan los significados que los estudiantes le atribuyen al signo igual o exploran estrategias de enseñanza para favorecer la comprensión de este signo en el ámbito escolar (por ejemplo, MOLINA; CASTRO; CASTRO, 2009; 
BURGELL; OCHOVIET, 2015; PARODI et. al., 2017), entre las que se destaca analizar igualdades numéricas en contextos no estándar de operaciones a la derecha o a ambos lados del signo igual. Se observa, además, una preocupación por develar la relación que existe entre las interpretaciones del signo igual y el desempeño de los estudiantes al resolver ecuaciones o tareas que involucran ecuaciones equivalentes (KNUTH et al., 2011; MATTHEWS et al., 2012). Estos trabajos, reportan que los alumnos de enseñanza primaria que definen el signo igual como un indicador de equivalencia tienen mayor probabilidad de resolver con éxito una ecuación del tipo $c+c+4=16$ o de identificar que $76+45-9=121-9$ es verdadero sabiendo que $76+45=121$ también lo es (MATTHEWS et al., 2012); del mismo modo que los alumnos de enseñanza media que definen el signo igual de esa manera tienden a resolver con éxito una ecuación del tipo $4 m+10=70$ o a identificar que las ecuaciones $2 m+15=31$ y $2 m+15-9=31-9$ son equivalentes, sin acudir a su resolución (KNUTH et al., 2011).

Con respecto a los polinomios, la investigación se ha focalizado en las dificultades de los estudiantes para reconocer expresiones algebraicas equivalentes y en la importancia de trabajar flexiblemente con estas expresiones (SOLARES; KIERAN, 2013). En particular, se reporta que los estudiantes tienden a convertir expresiones algebraicas en ecuaciones para obtener una representación que incluya un signo igual y un resultado (por ejemplo, TIROSH; EVEN; ROBINSON, 1998) y que presentan dificultades para aceptar una expresión algebraica como respuesta legítima a un problema ante la falta de un signo igual y un resultado (SFARD; LINCHEVSKI; 1994). Se exploran, asimismo, prácticas de enseñanza que pretenden abordar estas dificultades (TIROSH; EVEN; ROBINSON, 1998; KIERAN; SFARD, 1999); se discute la utilidad de calcular valores numéricos para validar la equivalencia de dos expresiones algebraicas (DEMBY, 1997) y se proponen estrategias para dotar de significado la simplificación de este tipo de expresiones (TABACH; FRIEDLANDER, 2017).

Se encuentra un solo estudio que relaciona específicamente las interpretaciones del signo igual y el trabajo de los estudiantes con este signo en un contexto algebraico de expresiones algebraicas (FYFE; MATTHEWS; AMSEL, 2017). Se reporta que los alumnos universitarios que participan en ese estudio y definen el signo igual en términos de equivalencia tienen mayor probabilidad de interpretar con éxito una expresión algebraica en la que no interviene este signo (por ejemplo, $4 c+3 b$ ). Se infiere, entonces, la necesidad de continuar realizando estudios de este tipo, pero en el ámbito de la enseñanza media, para explorar concretamente las interpretaciones del signo igual de los estudiantes en un contexto algebraico de polinomios. En esta línea se ubica el presente reporte de investigación. 


\section{Marco conceptual}

Este trabajo se inscribe en la noción de pensamiento relacional, que implica concebir a las expresiones aritméticas o algebraicas como totalidades, detectar o buscar relaciones entre ellas o entre sus términos y utilizar estas relaciones para resolver un problema, tomar una decisión o aprender más sobre una situación en particular (MOLINA, 2006). Este tipo de pensamiento es de carácter algebraico porque está ligado al estudio de las relaciones y las estructuras subyacentes, que son dos de los principales componentes de la perspectiva del álgebra considerada en este trabajo.

En este marco, se adopta como perspectiva teórica una clasificación de los significados del signo igual en contexto escolar (MOLINA; CASTRO; CASTRO, 2009). Esta clasificación fue elaborada a partir de los significados de este signo que son reconocidos y utilizados por la comunidad matemática, y por aquellos que son otorgados por los alumnos o son utilizados en los libros de texto de la matemática escolar.

1. Propuesta de actividad. El signo igual interviene en expresiones incompletas que incluyen una cadena de números o símbolos vinculados por símbolos operacionales solamente a la izquierda del signo. Ejemplo: $x(x+1)-3 x(x+5)=$.

2. Operador. El signo igual interviene en sentido unidireccional en igualdades que incluyen una cadena de operaciones a la izquierda del signo y el resultado de estas operaciones a la derecha. Ejemplo: $x(x-2)+3 x^{2}=4 x^{2}-2 x$.

3. Expresión de una acción. El signo igual separa una cadena de operaciones y el resultado de estas operaciones, admitiendo que operaciones y resultado estén indistintamente a la izquierda o a la derecha del signo. En este trabajo, se remitirá a este significado únicamente cuando el resultado esté a la izquierda del signo. Ejemplo: $2 x=x(x-2)-x^{2}+4 x$.

4. Separador. El signo igual separa los pasos realizados en la resolución de una actividad planteada en un contexto algebraico. Ejemplo: $x^{2}+1=x=x^{2}-x+1=0$.

5. Expresión de una equivalencia. El signo igual relaciona dos representaciones diferentes de un mismo objeto matemático. Se divide en tres acepciones:

5.1 Equivalencia numérica. Relaciona dos expresiones aritméticas que arrojan el mismo valor numérico. Ejemplo: $4+5=3+6$.

5.2 Equivalencia simbólica. Relaciona dos expresiones algebraicas que tienen el mismo valor numérico para todos los valores de la variable o las variables. Ejemplo: $x^{2}+2 x=x(x-2)$. 
5.3 Equivalencia por definición o por notación. Indica la equivalencia de dos expresiones numéricas o algebraicas por definición o por el significado de la notación utilizada. Ejemplo: $100 \mathrm{~cm}=1 \mathrm{~m}$.

6. Expresión de una equivalencia condicional. El signo igual expresa una equivalencia que es cierta para algún(os) valor(es) de la(s) variable(s), imponiendo que el conjunto solución de la ecuación correspondiente sea finito. Ejemplo: $x^{2}+4 x=5 x-6$.

7. Expresión de una relación funcional o de dependencia. El signo igual indica una relación de dependencia entre variables o parámetros. Ejemplo: $l=2^{\pi} r$.

8. Indicador de cierta conexión o correspondencia. El signo igual relaciona objetos no

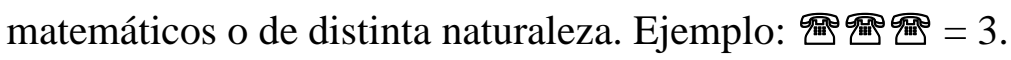

9. Indicador de una aproximación. El signo igual relaciona una expresión aritmética y una aproximación de su valor numérico. Ejemplo: $1 / 3=0,33$.

10. Definición de un objeto matemático. El signo igual define o asigna un nombre a una función u otro objeto matemático. Ejemplo: $f(x)=2 x+3$.

11. Asignación de un valor numérico. El signo igual asigna un valor numérico a un símbolo. Ejemplo: si $x=4$, ¿cuál es el valor numérico de $x-5$ ?

Esta clasificación se complementa con lo que varios autores denominan comprensión operacional, o comprensión relacional del signo igual (por ejemplo, KNUTH et al., 2011). Una comprensión operacional implica interpretar el signo igual como el indicador de un resultado o de cualquier otro modo que implique una lectura unidireccional (por ejemplo, como operador o propuesta de actividad), mientras que una comprensión relacional consiste en interpretar el signo igual como el indicador de una relación de equivalencia o de cualquier otro modo que implique una lectura bidireccional (por ejemplo, como expresión de una equivalencia). El Cuadro 1 clarifica la conjunción entre estos dos constructos.

\begin{tabular}{|l|c|c|}
\hline $\begin{array}{r}\text { Significado del } \\
\text { signo igual }\end{array}$ & Comprensión del \\
signo igual & Operacional & Relacional \\
\hline 1. Propuesta de actividad & $\mathrm{X}$ & \\
\hline 2. Operador & $\mathrm{X}$ & \\
\hline 3. Expresión de una acción & $\mathrm{X}$ & \\
\hline 4. Separador & & $\mathrm{X}$ \\
\hline 5. Expresión de una equivalencia condicional & & $\mathrm{X}$ \\
\hline 6. Expresión de una equivalencia & $\mathrm{X}$ & $\mathrm{X}$ \\
\hline 7. Expresión de una relación funcional o de dependencia & $\mathrm{X}$ & \\
\hline 8. Indicador de cierta conexión o correspondencia & $\mathrm{X}$ & \\
\hline 9. Indicador de una aproximación & $\mathrm{X}$ & \\
\hline 10. Definición de un objeto matemático & & \\
\hline 11. Asignación de un valor numérico & & \\
\hline
\end{tabular}

Cuadro 1 - Relación entre significados y tipos de comprensión del signo igual Fuente: elaboración propia 
En particular, el significado expresión de una equivalencia condicional está ligado a una comprensión relacional del signo igual, aunque no refiere a una relación de equivalencia, porque implica una lectura bidireccional del signo igual. Asimismo, el significado expresión de una acción está ligado a una comprensión operacional del signo igual, porque si bien interviene en expresiones en las que separa indistintamente una cadena de operaciones y el resultado de estas operaciones; en este trabajo, se hace referencia a este significado solamente cuando el resultado está a la izquierda del signo igual, implicando una lectura unidireccional. La conjunción entre la clasificación de significados (MOLINA; CASTRO; CASTRO, 2009) y los tipos de comprensión (KNUTH et al., 2011) del signo igual, así como estas consideraciones, resultaron operativas para mapear la conceptualización de este signo evidenciada por los dos estudiantes que se consideran para la realización de este reporte.

\section{Aspectos metodológicos}

Se aplicó un cuestionario a 26 estudiantes de un grupo de segundo año de enseñanza media (13-14 años) de un liceo privado de Montevideo, Uruguay. Una semana después se realizó una entrevista a cinco estudiantes, y un mes después se desarrollaron dos sesiones de trabajo con todos los estudiantes. El grupo estaba a cargo de uno de los investigadores y ya se habían abordado los temas de ecuaciones, polinomios y funciones. Específicamente, en polinomios, se había trabajado con valor numérico y las operaciones adición, sustracción y multiplicación. En este reporte, se informan hallazgos relativos a las respuestas que presentaron dos alumnos de este grupo a las cuatro preguntas del cuestionario que referían a polinomios o al significado explícito del signo igual, así como a las entrevistas que se realizaron con relación a estas cuatro preguntas.

\subsection{El cuestionario}

Se aplicó un cuestionario de dieciséis actividades en un contexto principalmente algebraico de ecuaciones y operaciones con polinomios. El tiempo máximo para responder este cuestionario fue de 120 minutos repartidos en una primera sesión de 80 minutos y una segunda sesión de 40 minutos. Los estudiantes recibieron y entregaron las actividades del cuestionario en forma alternada, para evitar nuevas correcciones que interfirieran en el análisis posterior. Se focaliza, a continuación, en el análisis a priori de las cuatro actividades de este cuestionario que exploran los significados que los alumnos le atribuyen explícitamente 
al signo igual (actividad I, Cuadro 2) y el trabajo de los alumnos con este signo en un contexto algebraico de polinomios (actividades II, III y IV).

Actividad I

Las siguientes actividades se refieren al símbolo que te presentamos a continuación:

a) ¿Cuál es el nombre que tiene este símbolo?

b) Explica con tus palabras cuál es el significado que tiene para ti este símbolo.

c) ¿En qué situaciones de clase has utilizado este símbolo? Ejemplifica, al menos, con tres situaciones distintas.

d) ¿Para qué se utiliza el = en cada una de las situaciones que presentaste?

Cuadro 2 - Actividad I del cuestionario aplicado a los estudiantes

Fuente: PARODI (2016)

En la actividad I se consulta el nombre y el significado del símbolo $=$, se solicita un mínimo de tres situaciones de clase en las que se hubiere utilizado este símbolo y la finalidad con que este símbolo se habría utilizado en cada situación. El propósito es indagar cuáles son los significados que los estudiantes le atribuyen explícitamente al signo igual, tanto al referirse al símbolo = como al plantear y describir las situaciones de clase solicitadas. En el análisis posterior, se considerará que un alumno presenta dos situaciones distintas si estas dejan entrever dos significados distintos del signo igual.

Si bien los alumnos cuentan con elementos para presentar ejemplos ligados a una comprensión relacional del signo igual, porque han incursionado en el estudio de las ecuaciones y los polinomios; no se descarta que tiendan a mostrar ejemplos que evidencien una interpretación operacional de este signo, influenciados por las prácticas de enseñanza que han recibido a lo largo de sus recorridos como estudiantes de enseñanza primaria. Esta actividad aporta al objetivo de investigación, porque aflorarán interpretaciones del signo igual que serán contrastadas con el trabajo de los alumnos con este signo en las actividades de contexto algebraico de polinomios. El Cuadro 3 muestra la Actividad II.

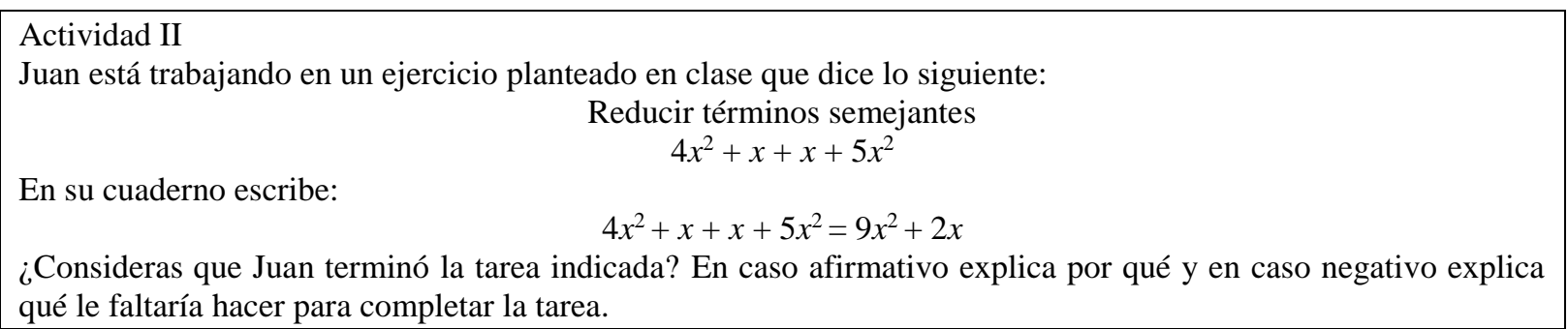

\section{Cuadro 3 - Actividad II del cuestionario aplicado a los estudiantes}

Fuente: PARODI (2016)

En la actividad II, a partir de un polinomio dado por un profesor y la expresión simplificada de ese polinomio propuesta por un estudiante llamado Juan, se pregunta si la tarea está terminada y se solicita una justificación. La consigna se redacta con la palabra reducir porque ese es el término que habitualmente se utiliza en el ámbito de la matemática escolar uruguaya para solicitar la simplificación de una expresión algebraica (en inglés, 
simplify). Una posibilidad es que los estudiantes consideren que la tarea está terminada porque Juan redujo términos semejantes y obtuvo un polinomio reducido equivalente al escrito por el profesor. En este caso, los alumnos podrán interpretar el signo igual como expresión de una equivalencia simbólica. Otra posibilidad es que los estudiantes cuestionen la equivalencia que escribió Juan porque a continuación del signo igual aparece un polinomio con más de un término y eso no es consistente con el uso operacional de este signo en contexto aritmético donde, en general, los sumandos aparecen a la izquierda y el resultado a la derecha del signo igual. Entonces, si afirman que Juan no terminó la tarea, podrá quedar de manifiesto una interpretación operacional del signo igual, ligada a la dificultad para aceptar que el objeto polinomio con más de un término puede ser el resultado de una operación.

En la actividad III (Cuadro 4) se plantean seis expresiones para que los estudiantes analicen y corrijan las que consideren falsas.

Actividad III

El profesor les planteó a sus estudiantes una tarea para trabajar con el tema polinomios. Te pedimos que la resuelvas.

Indica si cada una de las siguientes expresiones son verdaderas $(V)$ o falsas $(F)$. En aquellas que sean verdaderas explica por qué, y en las que sean falsas señala qué es lo que está mal y corrígelas para que sean verdaderas.
a) $x^{2}-9=(x+3)(x-3)$
c) $3 x^{2}+4 x^{2}=2 x^{2}+5 x^{2}$
e) $5+x=5+x$
b) $4 x=x+3 x$
d) $x=x$
f) $7 x+2 x=9 x$

Cuadro 4 - Actividad III del cuestionario aplicado a los estudiantes

Fuente: PARODI (2016)

Véase que, en las partes $a$ y $b$ se propone una equivalencia entre un polinomio reducido que está a la izquierda del signo igual y un polinomio no reducido que está a la derecha del signo igual, la diferencia radica en las operaciones involucradas en cada caso. En las partes $d$ y $e$ se propone una equivalencia entre dos polinomios idénticos, en la expresión $d$ no intervienen operaciones mientras que en la expresión $e$ interviene una suma a cada lado del signo igual. En las partes $c$ y $f$ se proponen otras dos equivalencias entre polinomios, la expresión $f$ tiene un solo término a la derecha del signo igual mientras que la expresión $c$ tiene dos. El propósito de esta actividad es indagar si los alumnos muestran evidencias de que pueden interpretar el signo igual como, por ejemplo, expresión de una acción (partes $a$ y $b$ ) o expresión de una equivalencia simbólica (partes $c, d, e$ y f). En particular, se podrá explorar si la presencia de un polinomio con más de un término a cada lado del signo igual favorece una interpretación relacional del signo igual.

En la actividad IV (Cuadro 5) se presenta un ejemplo de cómo simplificar polinomios y se plantean tres expresiones relacionadas con ese ejemplo para que los estudiantes analicen y corrijan las que consideren falsas. 
Actividad IV

La profesora escribe en el pizarrón que si reducimos monomios semejantes en la expresión $x+x+5+7$ obtenemos $2 x+12$. Es decir que: $x+x+5+7=2 x+12$.

Luego solicita a los alumnos que indiquen si lo escrito en cada renglón es Verdadero (V) o Falso (F). En caso de ser verdadero, les pide que expliquen por qué, y en caso de ser falso, les pide que indiquen lo que está mal.

$\begin{array}{lll}\text { a) }-5(x+x+5+7)=-5(2 x+12) & \text { b) } 2 x+12=x+x+5+7 & \text { c) } x+x+5+7-4=2 x+12-6\end{array}$

Te pedimos que resuelvas la tarea planteada.

Cuadro 5 - Actividad IV del cuestionario aplicado a los estudiantes

Fuente: PARODI (2016)

Una posibilidad es que los alumnos simplifiquen los dos polinomios que intervienen en cada expresión y comparen entre sí los polinomios así obtenidos. Otra posibilidad es que reconozcan que los dos polinomios equivalentes del ejemplo inicial han sido multiplicados por un mismo número (parte $a$ ) o han sido invertidos de lugar con respecto al signo igual (parte $b$ ) o han sido restados por números distintos (parte $c$ ). El propósito es explorar las estrategias que desarrollan los estudiantes para resolver esta tarea y contrastar esas estrategias con las interpretaciones del signo igual que han evidenciado los alumnos al resolver las otras tres tareas del cuestionario. En particular, se pretende indagar si los alumnos que explícitamente le han atribuido un significado relacional al signo igual son más propensos a desarrollar la segunda estrategia señalada, en comparación con aquellos estudiantes que explícitamente le hayan atribuido un significado operacional a este signo.

El diseño de estas actividades posibilita una exploración de una visión estructural de las expresiones, porque favorece la observación de características superficiales y ocultas (LIEBENBERG et al., 1998). Por ejemplo, los estudiantes, al mostrar y explicar situaciones de clase que involucren el signo igual, en la actividad I, podrán proponer expresiones del tipo $2+3=3+2$ e identificar que, en casos como este, se tiene el mismo resultado a cada lado del signo igual, que es una característica superficial, o que el orden de los sumandos no altera la suma, que es una característica oculta. Asimismo, al analizar la expresión de la actividad II, podrán apreciar que a izquierda y derecha del signo igual se tiene la misma cantidad de equis, que es una característica superficial, o que $x+x$ es igual a $2 x$ porque se cumple la propiedad distributiva de la multiplicación frente a la adición, que es una característica oculta.

Por otra parte, este diseño, permite focalizar en el desarrollo de un pensamiento relacional (MOLINA, 2006) porque las expresiones seleccionadas y el tipo de actividad que se solicita implican establecer relaciones entre y dentro de estas expresiones. Por ejemplo, los estudiantes, al analizar la expresión $c$ de la actividad III, en lugar de simplificar términos podrán detectar la compensación que se establece entre los términos involucrados para concluir que se trata de una expresión verdadera. Asimismo, al analizar la expresión $c$ de la 
actividad IV, podrán reconocer que la transformación aplicada a la expresión inicial no conserva la igualdad para concluir que se trata de una expresión falsa.

\subsection{Las entrevistas}

Luego de aplicar el cuestionario y realizar un análisis preliminar de los resultados obtenidos, se preseleccionaron siete estudiantes para realizarles una entrevista semiestructurada. La finalidad de estas entrevistas fue profundizar en algunas de las respuestas presentadas por estos estudiantes al cuestionario, ya sea por la originalidad de esas respuestas o por ser representativas de las respuestas dadas por otros estudiantes. Se seleccionaron estudiantes que, en su totalidad, abarcaran distintas maneras de pensar y responder el cuestionario. El día que se llevaron a cabo las entrevistas asistieron cinco de los siete alumnos preseleccionados. Las entrevistas fueron audio-grabadas.

\section{Resultados}

Las interpretaciones del signo igual que evidencian los alumnos al explicar o ejemplificar el significado de este signo (actividad I), así como el trabajo de los estudiantes con este signo en un contexto algebraico de polinomios (actividades II, III y IV), que son objeto de este reporte, se presentan a través de dos estudios de caso que son analizados en profundidad. Estos dos estudios de caso, permitirán ilustrar la diversidad de aspectos relativos a la problemática identificada, porque son representativos y esclarecedores del tipo de dificultades que se han encontrado en las respuestas de todos los estudiantes. Los casos seleccionados, en otras palabras, aportan suficientes evidencias para alcanzar el objetivo de este trabajo de investigación. En consecuencia, se procede al análisis exhaustivo de estos.

\subsection{El caso de Miguel}

En la actividad I, Miguel asegura que el símbolo = se llama es igual (parte a) y que este símbolo se utiliza cuando haces una cuenta, para saber cuánto da (parte b). Esta respuesta del alumno deja entrever una interpretación operacional del signo igual (por ejemplo, como operador o propuesta de actividad), porque manifiesta que es un signo que se escribe para indicar el resultado de una operación. Asimismo, Miguel señala o explica tres situaciones en las que se utiliza el signo igual: para hacer cuentas y saber el resultado, por 
ejemplo, $2+3=5$, en ecuaciones, para saber si son iguales y en geometría, para saber el lado de un triángulo, por ejemplo (partes $c$ y $d$ ).

Si bien en la primera situación, el estudiante vuelve a evidenciar una interpretación operacional del signo igual, en la segunda situación se refiere al proceso de verificación de una ecuación, que implica una comparación de valores numéricos a dos miembros y que es consistente con una interpretación del signo igual como expresión de una equivalencia condicional, porque deja entrever que la equivalencia dada por una ecuación no necesariamente es cierta para cualquier valor de la variable. En la tercera situación, el alumno se está refiriendo a expresiones del tipo $A B=6 \mathrm{~cm}$, que son habitualmente utilizadas en la matemática escolar para asignarle un valor numérico a la medida de un segmento; por lo tanto, deja al descubierto el significado de asignación de un valor numérico.

En la actividad II, que se muestra una situación en la que un profesor pide reducir términos semejantes en la expresión $4 x^{2}+x+x+5 x^{2}$ y un alumno llamado Juan escribe la expresión $4 x^{2}+x+x+5 x^{2}=9 x^{2}+2 x$; Miguel responde: No está terminada [la tarea] porque él [Juan] nunca reduce los términos sino que suma todo, ve cuánto da y entonces pone otra ecuación que dé lo mismo que la primera. Por un lado, cuando al justificar, Miguel afirma que Juan pone otra ecuación $\left[9 x^{2}+2 x\right]$ que dé lo mismo que la primera, si bien está utilizando el término ecuación en lugar de utilizar el término expresión o polinomio, su explicación da cuenta de una interpretación del signo igual como expresión de una equivalencia simbólica, porque hace mención a una igualdad entre dos expresiones: la que se ubica a la izquierda y a la derecha del signo igual. No obstante, esta interpretación del signo igual no le permite identificar que Juan terminó la tarea. Por otro lado, cuando al seguir justificando señala que Juan nunca reduce los términos, Miguel manifiesta la necesidad de continuar realizando operaciones hasta obtener un solo término a continuación del signo igual. Esto puede deberse a una interpretación del signo igual como operador que, en un contexto aritmético, implica plantear los sumandos a la izquierda y el resultado a la derecha del signo igual. El estudiante fue consultado sobre este asunto:

P: Explícame qué fue lo que pensaste en la actividad II.

M: Estaba viendo si son las mismas ecuaciones, no las estaba reduciendo.

$P:$ ¿Qué más habría que hacer para terminar con esa tarea?

M: Sacar esto, sacar toda la cuenta y poner... eh... reduciendo te da eso.

P: Mh... Yo te escribo acá lo que escribió Juan. ¿Por qué no terminó la tarea?

M: Porque él está diciendo que esta cuenta es igual a esta otra. No está reduciendo... No sé, para mí, está diciendo que son iguales, no está reduciendo.

$P: M h \ldots$. Ajá...

M: En verdad, está bien, redujo, pero yo lo tomé de otra forma... Como que estaba comparando, que no estaba reduciendo.

(Diálogo entre profesor-investigador y Miguel, 2016). 
Miguel insiste en que Juan no terminó la tarea, aunque sobre el final de la entrevista deja entrever lo contrario. Una posibilidad, es que el alumno continúe presentando dificultades para aceptar un polinomio con más de un término a la derecha del signo igual porque en otros contextos ha utilizado este signo en su carácter de operador. Otra posibilidad, que cobra fuerza cuando agrega que Juan está diciendo que [los polinomios] son iguales pero no está reduciendo, es que su respuesta esté influenciada por una interpretación de la palabra reducir como invitación a obtener un polinomio con menor cantidad de términos.

En la actividad III, que se proponen seis expresiones para analizar, Miguel sostiene que la expresión $x^{2}-9=(x+3)(x-3)$ es verdadera porque si hacés $3 x \cdot-3 x=-9 x^{2}$ (parte $a$ ) y que la expresión $4 x=x+3 x$ es verdadera porque $3 x+x$ te da $4 x$ (parte $b$ ). En el primer caso, que simplifica términos que no son semejantes (por ejemplo, $x+3$ lo simplifica a $3 x$ y $x^{2}-9$ a $9 x^{2}$ ), el producto que está a la derecha del signo igual le da como resultado el polinomio que está a la izquierda del signo y eso lo conduce a aceptar la expresión planteada. En ambos casos, el estudiante interpreta el signo igual como expresión de una acción, porque entiende que a la derecha del signo se planteó una operación y a la izquierda se planteó un posible resultado de esa operación. Esto, se fundamenta en el uso de la palabra $d a$ en $3 x+x$ te da $4 x$.

En esta actividad, Miguel también señala que la expresión $x=x$ es verdadera porque no hay suma y son los mismos números (parte $d$ ) y que la expresión $5+x=5+x$ es verdadera porque si hacés la cuenta de los dos lados te da lo mismo: $5+x=6 x$ y $5+x=6 x$ (parte $e$ ). En ambos casos, Miguel interpreta el signo igual como expresión de una equivalencia simbólica, porque hace alusión al resultado obtenido a cada lado del signo igual. No obstante, cuando al intentar simplificar los polinomios que intervienen en la parte $e$, escribe que $5+x=6 x$, el alumno interpreta el signo igual como operador porque lo utiliza para indicar que el resultado de la operación $5+x$ es $6 x$. Miguel simplifica términos que no son semejantes. Esta manera de proceder, que se ha observado en varias de las respuestas de Miguel al cuestionario, se relaciona con la necesidad de visualizar un solo término a continuación del signo igual y está ligada a una interpretación operacional del signo igual en contexto aritmético.

Por otra parte, Miguel señala que la expresión $7 x+2 x=9 x$ es verdadera porque $7 x+$ $2 x=9 x$ (parte $f$ ) y que la expresión $3 x^{2}+4 x^{2}=2 x^{2}+5 x^{2}$ es verdadera porque te da lo mismo: $3 x^{2}+4 x^{2}=7 x^{2}$ y $2 x^{2}+5 x^{2}=7 x^{2}$ (parte $c$ ). En el primer caso, cuando se limita a reescribir la expresión planteada en la actividad: $7 x+2 x=9 x$, está visualizando que $9 x$ es el resultado de $7 x+2 x$ o que $7 x+2 x$ y $9 x$ son dos maneras diferentes de expresar lo mismo. En el segundo caso, cuando al justificar simplifica términos semejantes y compara el monomio que se 
obtiene a cada lado del signo igual, está interpretando este signo como expresión de una equivalencia simbólica, porque reconoce que se obtiene el mismo resultado a cada lado.

No obstante, cuando al simplificar los polinomios que intervienen en la expresión escribe $3 x^{2}+4 x^{2}=7 x^{2}$ y $2 x^{2}+5 x^{2}=7 x^{2}$, da cuenta de una interpretación del signo igual como operador, porque lo utiliza para indicar que el resultado de $3 x^{2}+4 x^{2}$ es $7 x^{2}$ y que el resultado de $2 x^{2}+5 x^{2}$ es $7 x^{2}$. Esto muestra que distintos usos del signo igual conviven en un mismo estudiante y que puede utilizarlos en forma flexible de acuerdo con lo que desea expresar en cada caso, así como también, que la presencia de un polinomio con términos semejantes a ambos lados del signo igual puede favorecer una interpretación relacional del signo igual.

En la actividad IV, que a partir de la expresión $x+x+5+7=2 x+12$ se plantean tres expresiones para analizar, Miguel contesta que $-5(x+x+5+7)=-5(2 x+12)$ es verdadera y para justificar plantea que $5+7=12, x+x=2 x$ y agrega que si sumás te da lo mismo del otro lado y si lo multiplicas por -5 de los dos lados te da lo mismo (parte a). Cuando al justificar, señala que $5+7=12$ y que $x+x=2 x$, deja entrever una interpretación del signo igual como operador, porque lo utiliza para indicar el resultado de una operación entre números o una simplificación. No obstante, cuando agrega que si lo multiplicas por -5 de los dos lados te da lo mismo, en referencia al número por el que fueron multiplicados los dos polinomios de la consigna, evidencia una interpretación del signo igual como expresión de una equivalencia simbólica, porque reconoce que a cada lado de este signo se obtiene el mismo resultado.

Esta respuesta del alumno muestra, además, la emergencia de pensamiento relacional (MOLINA, 2006), porque establece relaciones entre y dentro de las expresiones al reconocer una transformación que conserva la igualdad. Asimismo, se evidencia que la adopción de un uso del signo igual en particular no desplaza los otros usos de este signo: por ejemplo, un entendimiento del signo igual como expresión de una equivalencia simbólica no impide que el alumno siga viéndolo como operador.

En esta actividad, Miguel también contesta que la expresión $2 x+12=x+x+5+7$ es verdadera porque es la misma que la que hizo la profesora, solo que cambió de lado los términos (parte $b$ ) y que la expresión $x+x+5+7-4=2 x+12-6$ es falsa porque $2 x+12-$ $6=6, x+x+5+7-4=8$ y lo que está mal es el -6 y el -4 , si los sacás o ponés el mismo número te da bien la ecuación (parte $c$ ). En el primer caso, en vez de simplificar y comparar los polinomios involucrados en la expresión planteada, que sería consistente con pensar en la definición de polinomios equivalentes, el alumno aplica implícitamente una propiedad equivalente a esa definición: si se invierten de lugar dos polinomios equivalentes respecto del signo igual, entonces, siguen siendo equivalentes. 
En otras palabras, el alumno reconoce una característica oculta (LIEBENBERG et al., 1998) de la expresión que analiza y la toma en cuenta para justificar su respuesta. En el segundo caso, si bien vuelve a confundir expresión con ecuación, el alumno está interpretando el signo igual como expresión de una equivalencia simbólica, porque inspecciona globalmente la expresión e identifica que se restó un número distinto a cada lado del signo igual de la expresión inicial. No obstante, al intentar simplificar los polinomios que intervienen en la expresión, Miguel escribe que $2 x+12-6=6$ y que $2 x+12-4=8$, evidenciando un uso operacional del signo igual. Además, omite la variable que figura en cada polinomio y se limita a simplificar los términos numéricos, haciendo caso omiso de lo desconocido (KIERAN, 1984).

\subsection{El caso de Gerónimo}

En la actividad I, Gerónimo asegura que el símbolo = se llama igual (parte a) y que todo lo que esté a sus lados, finalmente, luego de hacer las cuentas, son iguales, tienen el mismo valor (parte $b$ ). Esta respuesta del estudiante evidencia una interpretación relacional del signo igual (por ejemplo, como expresión de una equivalencia numérica o simbólica) porque deja entrever que este signo relaciona expresiones que arrojan el mismo valor numérico. Asimismo, Gerónimo ejemplifica y explica tres situaciones en las que se utiliza el signo igual: en $3 x=9$, para representar cuánto vale $3 x$ y poder hallar $x$, en $f(x)=6 x-3$, para explicar que la función correspondiente al gráfico $f(x)$ es $6 x-3$ y en $5+5=10$, para expresar que lo de la derecha del signo es igual o equivalente a lo del otro lado del signo (partes $c$ y $d$ ). En la primera situación, el estudiante manifiesta una interpretación del signo igual como expresión de una equivalencia condicional, porque deja entrever que la ecuación que propone se transforma en una igualdad numérica para un valor particular de la variable. En la segunda situación, Gerónimo está interpretando el signo igual como definición de un objeto matemático, porque lo utiliza para asignarle un nombre a una función. En la tercera situación, si bien presenta un ejemplo numérico en un contexto de operaciones igual respuesta, el alumno interpreta el signo igual como expresión de una equivalencia numérica porque hace referencia al valor numérico obtenido a cada lado del signo igual.

En la actividad II, que se muestra una situación en la que un profesor pide reducir términos semejantes en la expresión $4 x^{2}+x+x+5 x^{2}$ y un alumno llamado Juan escribe la expresión $4 x^{2}+x+x+5 x^{2}=9 x^{2}+2 x$; Gerónimo responde: Creo que está en lo correcto, ya que además de reducirlos bien, están perfectamente ordenados de mayor exponente a menor 
exponente. Por un lado, Gerónimo da cuenta de que, al simplificar un polinomio que se encuentra a la izquierda del signo igual, es posible obtener un polinomio con más de un término a la derecha del signo igual. Esto, es coherente, por ejemplo, con el significado que él le atribuyó al símbolo = en la actividad I del cuestionario. Por otro lado, cuando dice que están perfectamente ordenados de mayor exponente a menor exponente, deja entrever que simplificar un polinomio también implica ordenar el polinomio reducido que se obtiene.

En la actividad III, Gerónimo sostiene que las expresiones $a$ y $b$ son verdaderas. En la parte $a$, cuando dice que está bien ya que quedaría $x^{2}+3 x-3 x-9$ y abajo escribe $x^{2}-9$ (Figura 1), deja entrever que el resultado de la operación $(x+3)(x-3)$ es $x^{2}-9$. En la parte $b$, cuando escribe $x+3 x=x+x+x+x=4 x$, lo hace para señalar que el resultado de la operación $x+3 x$ es $4 x$. Desde la perspectiva del estudiante, las expresiones que aparecen a la derecha del signo igual plantean una operación y las expresiones de la izquierda son entendidas como los resultados de esas operaciones. Entonces, en ambos casos, el alumno está interpretando el signo igual como expresión de una acción. En particular, se observa que el alumno utiliza el signo igual en ese sentido al realizar la operación $x+3 x$, cuando escribe $x+$ $3 x=x+x+x+x$.

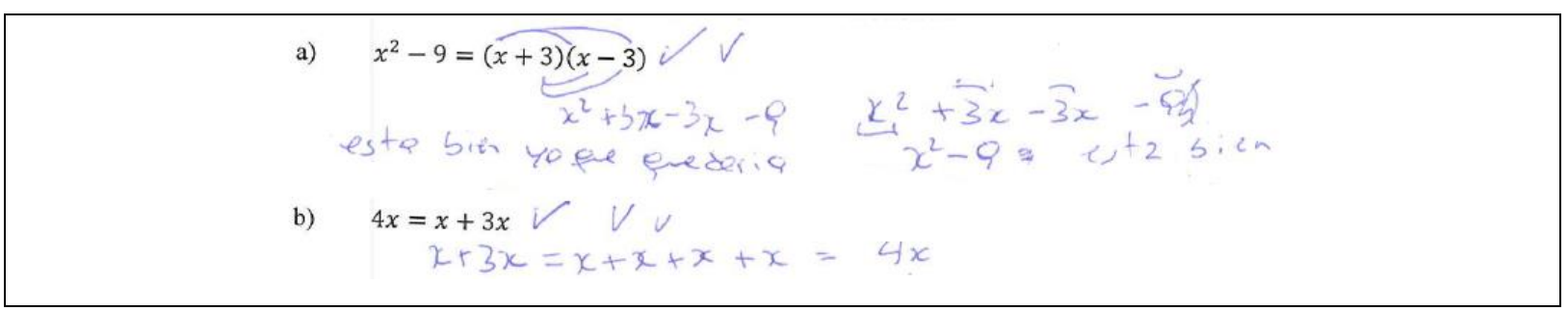

Figura 1 - Respuesta de Gerónimo a la actividad III $a$ y III $b$ del cuestionario Fuente: producción del alumno Gerónimo

En esta actividad, Gerónimo también señala que la expresión $x=x$ es verdadera porque al estar el mismo número a cada lado del igual, dan lo mismo (parte $d$ ) y que la expresión $5+x=5+x$ es verdadera porque al estar la misma cuenta a cada lado claramente son iguales (parte $e$ ). En el primer caso, el alumno reconoce que la variable toma un solo valor por vez cuando dice que está el mismo número a cada lado del igual. En ambos casos, el estudiante está interpretando el signo igual como expresión de una equivalencia simbólica porque entiende que se obtiene el mismo valor numérico a cada lado del signo para cualquier valor de la variable. Gerónimo establece una comparación entre los polinomios involucrados que evidencia una comprensión relacional del signo igual: la misma cuenta a cada lado.

Por otra parte, Gerónimo señala que la expresión $3 x^{2}+4 x^{2}=2 x^{2}+5 x^{2}$ es verdadera porque las sumas de ambos lados del igual dan $7 x^{2}$ (parte $c$ ) y que la expresión $7 x+2 x=9 x$ es verdadera porque ambos lados dan $9 x$ (parte f). En ambos casos, el estudiante está 
interpretando el signo igual como expresión de una equivalencia simbólica, porque hace alusión al resultado que se obtiene a cada lado del signo igual. La presencia de un polinomio con un solo término a la derecha del signo igual (parte $f$ ) no impide que Gerónimo interprete el signo igual en forma relacional. Además, el estudiante pasa por las expresiones $9 x=9 x$ (parte $c$ ) y $7 x^{2}=7 x^{2}$ (parte $f$ ) para justificar sus respuestas, mostrando la necesidad de operar con los polinomios hasta obtener una misma expresión a cada lado del signo igual.

En la actividad IV, que a partir de la expresión $x+x+5+7=2 x+12$ se plantean otras tres expresiones para analizar, Gerónimo sostiene que las expresiones $a$ y $b$ son verdaderas y que la expresión $c$ es falsa. En los tres casos, el estudiante realiza operaciones hasta obtener un polinomio ordenado y reducido a cada lado del signo igual para analizar la validez de cada expresión. Asimismo, el alumno, para complementar los cálculos realizados, presenta una explicación verbal que relaciona la equivalencia dada en la consigna con la expresión analizada en cada parte de la actividad: son iguales, ya que al ser ambos multiplicados por el mismo número, cambian su valor pero siguen siendo equivalentes (parte a), es lo que propuso la profesora, además, luego de sumar dan igual (parte b) y son diferentes porque antes eran iguales, mientras que ahora se le resta 4 al de la izquierda y 6 al de la derecha (parte $c$ ).

Gerónimo reconoce que al multiplicar dos polinomios equivalentes por un mismo número o al intercambiar estos dos polinomios de lugar con respecto al signo igual se mantiene la equivalencia, así como al sumar un número distinto a dos polinomios equivalentes dejan de ser equivalentes. Estas explicaciones evidencian una interpretación del signo igual como expresión de una equivalencia simbólica, así como la emergencia de pensamiento relacional (MOLINA, 2006) y una atención explícita a las características ocultas de las expresiones analizadas (LIEBENBERG et al., 1998). No obstante, Gerónimo necesita operar para comparar los polinomios resultantes e inferir la validez de cada expresión.

\section{Discusión}

Por un lado, Miguel, que evidencia una comprensión mayoritariamente operacional del signo igual al explicar y ejemplificar el significado del símbolo $=($ actividad $\mathrm{I})$, deja entrever que ante la solicitud de simplificar un polinomio se debe obtener un solo término a continuación del signo igual (actividad II). Esta dificultad para aceptar el objeto polinomio con más de un término como resultado de una simplificación está ligada a una interpretación del signo igual como operador o propuesta de actividad en contexto aritmético. Esta interpretación del signo igual, a su vez, es especialmente perjudicial cuando conduce al 
estudiante a simplificar términos que no son semejantes con tal de obtener un solo término a continuación del signo igual: por ejemplo, $5+x=6 x$ (actividad III $e$ ).

No obstante, la interpretación operacional del signo igual que manifiesta Miguel en varias de sus respuestas, no le impide entender el signo igual como expresión de una equivalencia simbólica y mostrar indicios de desarrollo de pensamiento relacional (por ejemplo, actividades IV $a$ y IVc), así como atender características ocultas de las expresiones que analiza (actividad IV $b$ ). Esto muestra que distintos usos del signo igual conviven en un mismo estudiante y que puede utilizarlos en forma flexible de acuerdo con lo que desea expresar en cada caso, del mismo modo que la adopción de un significado del signo igual no desplaza los otros significados de este signo que el estudiante ya conoce o ha manifestado antes. El trabajo de Miguel también da muestras de que el análisis de una expresión en la que intervienen polinomios con términos semejantes a cada lado del signo igual puede favorecer una interpretación relacional del signo igual (actividad IIIc).

Por otro lado, Gerónimo, que evidencia una comprensión mayoritariamente relacional del signo igual al explicar y ejemplificar el significado del símbolo = (actividad $\mathrm{I})$, tiende a interpretar este signo como expresión de una equivalencia simbólica en un contexto algebraico de polinomios (actividades II, III $d$ y III $c$, entre otras), excepto cuando la afirmación incluye un polinomio reducido a la izquierda del signo igual, caso en el que suele interpretarlo como expresión de una acción (actividades III $a$ y III $b$ ). En general, el estudiante realiza simplificaciones para luego comparar los polinomios obtenidos a cada lado del signo igual y decidir acerca de la validez de cada expresión. Asimismo, el alumno reconoce que la equivalencia entre dos polinomios se conserva si estos polinomios son multiplicados por un mismo número o se invierten de lugar con respecto al signo igual, mostrando indicios de desarrollo de pensamiento relacional y una atención explícita a las características ocultas de las expresiones que analiza, aunque prefiere realizar cálculos para complementar cada una de sus respuestas (actividad IV). Esto deja entrever que el alumno acude a distintos usos del signo igual en forma simultánea con el objetivo de dar respuesta a una situación problemática y que este pasaje por distintos usos del signo igual fortalece el entendimiento de cada uno de estos.

En conjunto, los resultados de este estudio ponen de manifiesto que hay interpretaciones del signo igual que enriquecen el trabajo de los estudiantes en un contexto algebraico de polinomios y otras que lo obstaculizan. Por ejemplo, una interpretación del signo igual como operador, puede conducir a los estudiantes a simplificar términos de un polinomio que no son semejantes con tal de obtener un solo término a continuación del signo igual (Miguel, actividad II). Esto se debe a que, en contexto aritmético, una interpretación del 
signo igual como operador o propuesta de actividad, implica que a continuación de este signo solo aparezca un número como resultado de las operaciones planteadas a la izquierda. Entonces, en contexto algebraico, esta visión operacional del signo igual obstaculiza la aceptación de un polinomio con más de un término como resultado de una simplificación.

Del mismo modo, una interpretación relacional del signo igual, puede conducir a los alumnos a reconocer que, por ejemplo, si dos polinomios son equivalentes y se multiplican por un mismo número, entonces, los polinomios resultantes también son equivalentes (Gerónimo, actividad IV). Es decir, la interpretación del signo igual como expresión de una equivalencia aparece ligada al reconocimiento de las transformaciones que conservan la igualdad y favorece una emergencia de pensamiento relacional, a la vez que posibilita una atención explícita a las características ocultas de las expresiones.

Por otra parte, el trabajo de los estudiantes en un contexto algebraico de polinomios también puede abonar a la interpretación del signo igual. Por ejemplo, el análisis de expresiones en las que intervienen polinomios equivalentes con términos semejantes a cada lado del signo igual, favorece una interpretación del signo igual como expresión de una equivalencia simbólica (Miguel, actividad III).

\section{Conclusiones}

En este reporte se presentaron dos estudios de caso para evidenciar la relación entre los significados que se le atribuyen explícitamente al signo igual y el trabajo con este signo en un contexto algebraico de polinomios. Los hallazgos obtenidos, con respecto a los dos casos reportados, ponen en evidencia una relación dialéctica entre los significados que se atribuyen explícitamente al signo igual y el trabajo con este signo en un contexto de polinomios.

Es decir, un conocimiento relacional del signo igual facilita el entendimiento de las operaciones con polinomios, que es consistente con lo reportado en la literatura acerca de la importancia de comprender este signo para incursionar en el estudio del álgebra (KNUTH et al., 2011; FYFE; MATTHEWS; AMSEL, 2017; entre otros), pero, adicionalmente, los resultados de esta investigación también evidencian que el trabajo con polinomios favorece la comprensión relacional del signo igual. En particular, así como la comprensión del signo igual como indicador de equivalencia favorece la emergencia de pensamiento relacional y la exploración de una visión estructural de las expresiones, este reporte muestra que el contexto algebraico de polinomios favorece y habilita esa posibilidad. 
La mayoría de los estudios relativos al signo igual afirman que es necesario que el alumno comprenda este signo de manera relacional como condición previa para iniciarse con éxito en el estudio del álgebra (por ejemplo, KIERAN, 1992; KNUTH et al., 2011; MATTHEWS et al., 2012; FYFE; MATTHEWS; AMSEL, 2017). Sin embargo, este estudio pone de relieve que no se puede asegurar que los estudiantes necesitan comprender primero el significado relacional del signo igual para luego adentrarse en el estudio de los polinomios, sino que el contexto algebraico de los polinomios va a originar y promover la comprensión de nuevos significados, así como el fortalecimiento de otros significados, ya bien conocidos por los estudiantes. Estos hallazgos discuten la idea de secuencialidad del aprendizaje, en el sentido que la transición entre los significados del signo igual retroalimenta la comprensión de cada uno de estos y la comprensión de este signo se presenta como algo cíclico y no lineal.

El surgimiento de la dialéctica identificada está ligado al diseño de las actividades del cuestionario, que promueve un uso diversificado de significados del signo igual. Entonces, como recomendación didáctica, se sugiere a los profesores atender explícitamente los significados del signo igual como objeto de enseñanza, no solo en forma previa al estudio del álgebra, en general, y de los polinomios, en particular, sino también, desde el propio contexto algebraico de polinomios. Esta atención puede darse a través del diseño de actividades que pongan en juego, por ejemplo: el análisis de cómo, por qué y para qué se usa el signo igual en contexto aritmético y algebraico, la discusión de producciones de estudiantes que requieran movilizar un uso operacional y relacional del signo igual, la lectura bidireccional de las igualdades obtenidas al operar con polinomios y actividades que requieran argumentar si dos o más escrituras distintas pueden corresponder a un mismo polinomio.

En suma, el contexto algebraico de polinomios es una oportunidad para introducir nuevos significados del signo igual y fortalecer, asimismo, aquellos significados de este signo que los estudiantes ya conocen o ya han estudiado a lo largo de su trayectoria escolar.

\section{Referencias}

BURGELL, F.; OCHOVIET, C. Significados del signo igual y aspectos de su enseñanza. Un estudio realizado con estudiantes de primer año de enseñanza secundaria y sus profesores. Enseñanza de las Ciencias, Barcelona, v. 33, n. 3, p. 77-98, jul. 2015.

DEMBY, A. Algebraic procedures used by 13-to-15-year-olds. Educational studies in mathematics, Utrecht, v. 33, n. 1, p. 45-70, jun. 1997.

FYFE, R.; MATTHEWS, P.; AMSEL, E. College students' knowledge of the equal sign and its relation to solving equations. En: THE ANNUAL MEETING OF PME-NA, 39., 2017, Indianapolis.

Proceedings... Indianapolis: Hoosier AMTE, 2017. p 279-283. 
KIERAN, C. A comparison between novice and more-expert algebra students on tasks dealing with the equivalence of equations. En: THE ANNUAL MEETING OF PME-NA, 6., 1984, Madison. Proceedings... Madison: Hoosier AMTE, 1984. p. 83-91.

KIERAN, C. The Learning and Teaching of School Algebra. En: GROUWS, D. (ed.). Handbook of research on mathematics teaching and learning: A project of the NCTM. New York: Macmillan, 1992. p. 390-419.

KIERAN, C.; SFARD, A. Seeing through symbols: The case of equivalent expressions. Focus on learning problems in mathematics, Massachusetts, v. 21, n. 1, p. 1-17, ene. 1999.

KNUTH, E.; ALIBALI, M.; MCNEIL, N.; WEINBERG, A.; STEPHENS, A. Middle school students' understanding of core algebraic concepts: Equivalence y variable. En: CAI, J.; KNUTH, E. (ed.). Early algebraization: Advances in Mathematics Education. Berlin: Springer, 2011. p. 259-276.

LIEBENBERG, R.; LINCHEVSKI, L.; OLIVIER, A.; SASMAN, M. Laying the foundation for algebra: Developing an understanding of structure. En: THE ANNUAL CONGRESS OF AMESA, 4., 1998, Polokwane. Proceedings... Polokwane: AMESA, 1998. p. 1-5.

MATTHEWS, P.; RITTLE-JOHNSON, B.; McELDOON, K.; TAYLOR, R. Measure for measure: What combining diverse measures reveals about children's understanding of the equal sign as an indicator of mathematical equality. Journal for Research in Mathematics Education, Reston, v. 43, n. 3, p. 316-350, may. 2012.

MOLINA, M. Desarrollo de pensamiento relacional y comprensión del signo igual por alumnos de tercero de educación primaria. 2006. 713 f. Tesis (Doctorado en Didáctica de la Matemática) Universidad de Granada, Granada, 2006.

MOLINA, M.; CASTRO, E.; CASTRO, E. Elementary students understanding of the equal sign in number sentences. Electronic Journal of Research in Educational Psychology, Almería, v. 17, n. 1, p. 341-368, abr. 2009.

NATIONAL COUNCIL OF TEACHERS OF MATHEMATICS. Principals and Standards for School Mathematics. USA: NCTM, 2008.

PARODI, S. Significados del signo de igual en la entrada al algebra: un estudio de casos con estudiantes de segundo año de enseñanza secundaria. 2016. 303 f. Tesis (Maestría en Ciencias en Matemática Educativa) - CICATA, IPN, México DF, 2016.

PARODI, S.; OCHOVIET, C.; LEZAMA, J. La comprensión del signo de igual en la entrada al álgebra: el diseño de tareas y la conversación en la clase de matemática. Enseñanza de las Ciencias, Barcelona, v. 35, n. 3, p. 51-67, nov. 2017.

RADFORD, L. Grade 2 students' non-symbolic algebraic thinking. En: CAI, J.; KNUTH, E. (ed.). Early algebraization: Advances in Mathematics Education. Berlin: Springer, 2011. p. 303-322.

SFARD, A.; LINCHEVSKI, L. The gains and the pitfalls of reification. The case of Algebra. Educational Studies in Mathematics, Utrecht, v. 26, n. 1, p. 191-228, mar. 1994.

SOLARES, A.; KIERAN, C. Articulating syntactic and numeric perspectives on equivalence. Educational Studies in Mathematics, Utrecht, v. 84, n. 1, p. 115-148, set. 2013.

TABACH, M.; FRIEDLANDER, A. Algebraic procedures and creative thinking. ZDM, Berlin, v. 49, n. 1, p. 53-63, mar. 2017. 
TIROSH, D.; EVEN, R.; ROBINSON, N. Simplifying algebraic expressions: Teacher awareness and teaching approaches. Educational Studies in Mathematics, Utrecht, v. 35, n. 1, p. 51-64, ene. 1998.

Submetido em 19 de Dezembro de 2019. Aprovado em 07 de Julho de 2020. 